\title{
Birth outcome in women with breast cancer
}

\author{
V Langagergaard ${ }^{*, 1,2}$, M Gislum', MV Skriver', B Nørgård', TL Lash', KJ Rothman ${ }^{3}$ and HT Sørensen ${ }^{1,3}$ \\ 'Department of Clinical Epidemiology, Aarhus University Hospital, Ole Worms Allé 150, DK-8000, Aarhus C, Denmark; ${ }^{2}$ Department of Epidemiology, \\ Institute of Public Health, Aarhus University, Vennelyst Boulevard 6, DK-8000, Aarhus C, Denmark; ${ }^{3}$ Department of Epidemiology, School of Public \\ Health, Boston University, 715 Albany Street, TE3, Boston, MA 021 I8, USA
}

We investigated whether maternal breast cancer affects birth outcome in a nationwide cohort study of 695 births from 1973 to 2002 of women with breast cancer with respect to preterm birth, low birth weight at term, stillbirth and congenital abnormalities as well as mean birth weight, compared with the outcomes of 33443 births from unaffected mothers. There was no excess risk of adverse birth outcome for the 216 newborns of women with breast cancer before pregnancy. Stratification by mother's treatment did not change the results. For 37 newborns of women diagnosed during pregnancy, the prevalence ratio (PR) of preterm birth was 8.1 (95\% confidence interval (Cl): 3.8-17). However, 10 of the 12 preterm deliveries among these women were elective early deliveries. Among 442 births of women diagnosed in the 2 years from time of delivery, the PR of preterm birth was 1.4 (95\% Cl: I.0-2.0), and the PR of low birth weight at term for boys was 2.9 (95\% Cl: 1.3-6.3). Overall, our results are reassuring regarding the risks of adverse birth outcome for breast cancer patients.

British Journal of Cancer (2006) 94, I42- 146. doi:I0.1038/sj.bjc.6602878 www.bjcancer.com

Published online 22 November 2005

(c) 2006 Cancer Research UK

Keywords: epidemiology; breast cancer; birth outcome; cohort study

In western countries many women postpone childbearing for personal or professional reasons (Dow et al, 1994), which both increases their risk for breast cancer (Kelsey et al, 1993) and reduces the period between giving birth and breast cancer diagnosis. In the future, therefore, more breast cancer patients will have recently given birth, been pregnant concurrent with their diagnosis, or not yet started their childbearing at the time of their diagnosis.

Biological mechanisms related to the cancer or its treatment may impact foetal growth, development, and teratogenesis (Zemlickis et al, 1992; Zhu et al, 2002). However, the epidemiologic evidence of the effect of breast cancer on birth outcome is limited. The few studies of women who were diagnosed with breast cancer before pregnancy have focused on maternal prognosis (Ribeiro et al, 1986; Sutton et al, 1990; Dow et al, 1994; Malamos et al, 1996; Kroman et al, 1997; Velentgas et al, 1999; Blakely et al, 2003). Thus, no population-based cohort study of birth outcome in women diagnosed with breast cancer before pregnancy has been published. Cohorts without control groups including between four and 121 women (Ribeiro et al, 1986; Daly and Donnellan, 1992; Berry et al, 1999; Giacalone et al, 1999; Kuerer et al, 2002; Ring et al, 2005) have shown that the majority of women who are diagnosed with breast cancer during or shortly after pregnancy give birth to healthy children. Two controlled studies, however, suggested an increased risk of preterm birth and low birth weight for offspring of these women (Zemlickis et al, 1992; Smith et al, 2001). Therefore, within a cohort study, we examined birth

*Correspondence: Dr $\vee$ Langagergaard;

E-mail: vl@dce.au.dk

Received 22 August 2005; revised 3 October 2005; accepted 26 October 2005; published online 22 November 2005 outcome in all women diagnosed with breast cancer in Denmark from 1943 to 2002, compared with women without cancer.

\section{MATERIAL AND METHODS}

\section{Study population}

We conducted this nationwide cohort study based on all Danish women who were diagnosed with breast cancer from January 1, 1943 through December 31, 2002, and who gave birth from January 1, 1973 through December 31, 2002. Women were included if they were diagnosed at any time before pregnancy, during the pregnancy, or until 2 years post partum. Their birth outcome was compared with the outcome in a comparison cohort selected from other births in Denmark. We restricted all analyses to singleton births only, and each pregnancy was included in the analyses as an independent event.

Breast cancer cohort Women with breast cancer were identified from the Danish Cancer Registry, which has kept records of all incident cases of cancer in Denmark since 1943, classified according to the International Classification of Diseases (ICD-7) (Storm et al, 1997). The records include the civil registration number, diagnosis, date of diagnosis, method of verification, extent of spread of the tumour at time of diagnosis, and treatment given within 4 months after diagnosis. We identified all women with a diagnosis of breast cancer (ICD-7 codes 170.0-170.5). We excluded all cases of 'Carcinoma in situ' and six cases of sarcoma involving the breast. Women with breast cancer were linked to the Danish Medical Birth Registry with data on all births in Denmark since January 1, 1973 (Kristensen et al, 1996) obtained from birth notifications, filled in by midwives (in Denmark all births, 
including home births, are attended by midwives). The main variables are the civil registration number of the mother and child, date and place of birth, gestational age, birth weight, and parity. Birth weights $\geqslant 7000 \mathrm{~g}$ probably reflected coding errors and were excluded from the analyses. Likewise, we excluded births registered without a gestational age or when this was less than 20 weeks or more than 44 weeks. Owing to a change in classification procedures in the Birth Registry in 1978, there was more missing data on gestational age for the years 1978-1981 than for other years (mean missing proportion, $21.7 \%$ for $1978-1981$, compared with $2.4 \%$ for the years $1973-1977$, and $0.7 \%$ for the years 1982 -2002). We identified 695 singleton births delivered by women in the breast cancer cohort.

Comparison cohort For each birth by a woman with breast cancer, 50 comparison births matched by month and year of birth, by county of mother's residence, and born to 50 different women, who were not diagnosed with any cancer before or during the pregnancy or until 2 years after the birth were selected from the Birth Registry. If fewer than 50 comparison births fulfilled the criteria, we used the available number of births. If more than 50 comparison births were eligible after matching, the subset of 50 was randomly selected. On average, 48 comparison births were selected for each exposed birth. Altogether, 33443 single births were selected for the comparison cohort.

Outcome data The data collected from the Birth Registry included preterm birth (birth before 37 completed weeks of pregnancy), low birth weight at term (birth weight $<2500 \mathrm{~g}$ with a gestational age $\geqslant 37$ completed weeks of pregnancy), stillbirth (delivery of a dead foetus at 28 completed weeks of gestation or later in pregnancy), male proportion of newborns, and birth weight. Data on potential confounders included maternal age, parity, gestational age, and calendar period of the birth. Data about congenital abnormalities (including chromosomal abnormalities) diagnosed during the first year after the birth were collected from the Danish Hospital Discharge Registry, established in 1977, with records of all discharges from Danish hospitals. The recorded information includes the civil registration number, dates of admission and discharge, and up to 20 discharge diagnoses, using the International Classification of Diseases (ICD-8 before 1994 and ICD-10 from 1994 onward (Andersen et al, 1999)). The codes for congenital abnormalities (including chromosomal abnormalities) were 740.00-759.99 in ICD-8 and Q0.00-Q99.9 in ICD-10. Diagnoses of congenital dislocation of the hip and undescended testis were excluded because of their poor validity (Larsen et al, 2003).

\section{Record linkage}

Linkage between registries was made by the civil registration number stored in the Danish Civil Registration System together with information on vital status, emigration, address, and nuclear family members' civil registration number since 1968 (Frank, 2000).

\section{Data analysis}

We classified the births of women with breast cancer according to time of cancer diagnosis in relation to pregnancy. Group 1 included the first birth after breast cancer delivered by women who were diagnosed at any time before pregnancy. Group 2 included the births delivered by women with a diagnosis of breast cancer during their pregnancy (i.e., diagnosed between the first day in the last menstruation until the date of birth). Group 3 included births delivered by women who were diagnosed with breast cancer after delivery (i.e., diagnosed between the day after the date of birth until 2 years later). If a woman gave birth more than once in this 2-year period, only the last birth before the cancer diagnosis was included. We computed the difference between the male proportion of newborns of women with breast cancer and that of newborns of matched comparison mothers with $95 \%$ confidence intervals $(95 \% \mathrm{CI})$ for these differences. We estimated the prevalence ratios (PR) using prevalence odds ratios and 95\% CI for preterm birth, low birth weight at term, stillbirth, and congenital abnormalities by logistic regression modelling. Stillborn children were excluded from the analyses of preterm birth, low birth weight at term, and congenital abnormalities. We adjusted for maternal age, parity, and calendar period of the birth. PRs for congenital abnormalities were additionally adjusted for gestational age. For births in Groups 1 and 3, we repeated the analyses in strata of boys and girls to examine if sex of the child modified the PR estimates. For births in Group 1, we evaluated whether treatment of the mother modified the PR estimates by repeating the analyses in strata of births of women treated with surgery alone and births of women who received other treatment (i.e., radiotherapy, chemotherapy, or endocrine therapy).

We used multivariate regression analysis to estimate differences in mean birth weight adjusted for maternal age, parity, gestational age, and calendar period of the birth. Stillborn children were excluded from this analysis.

All analyses used SAS software, version 8.2.

The study was approved by the Danish Data Protection Agency (record no. 2003-41-2833).

\section{RESULTS}

Descriptive data on Groups 1, 2 and 3 and their matched comparison births are shown in Table 1 . Of the 695 single births delivered by women with BC, 216 occurred in Group 1, 37 occurred in Group 2, and 442 occurred in Group 3. For Group 1, the median number of days from the time of diagnosis until pregnancy (i.e., the first day in the last menstruation) was 753 days (range: 3-5965 days). Of the 37 births in Group 2, one woman was diagnosed in the first trimester, five in the second, and 31 women were diagnosed in the third.

For births delivered by women in Group 3, the median number of days from date of the birth until date of cancer diagnosis was 417 days (range: $1-729$ days).

We evaluated the proportion of male newborns of women with breast cancer compared with that of newborns of unaffected mothers ( 50 vs $52 \%$, difference $=-2.2 \%$, $(95 \% \mathrm{CI}=-8.9 ; 4.5)$ for Group 1, 49 vs $52 \%$, difference $=-3.4 \%,(95 \% \mathrm{CI}=-20 ; 13)$ for Group 2, and 53 vs $51 \%$, difference $=2.5 \%,(95 \% \mathrm{CI}=-2.2 ; 7.2)$ for Group 3).

Table 2 shows the PRs for preterm birth, low birth weight at term, stillbirth and congenital abnormalities for newborns in Groups 1-3. There was no stillborn child among the births delivered by mothers with breast cancer. For births in Group 1, we found no increased odds of low birth weight at term or congenital abnormalities and no substantial increased odds of preterm birth. For births in Group 2, the odds of preterm birth increased by eight-fold $(\mathrm{PR}=8.1,95 \%$ $\mathrm{CI}=3.8-17)$. However, 10 of the 12 preterm deliveries among the women with breast cancer were elective preterm deliveries. As a result of the small number of outcome events, effect estimates for Group 2 were imprecise. For Group 3 the PR of preterm birth was $1.4(95 \% \mathrm{CI}=1.0-2.0)$. For low birth weight at term the PR was $1.4(95 \% \mathrm{CI}=0.7-2.8)$. There was no increased prevalence of congenital abnormalities. We found no clusters of congenital abnormalities in any specific organ system and there was only one case with a chromosomal abnormality (data not shown). Stratification according to sex of the offspring in Groups 1 and 3 did not change the overall effect estimates substantially (data not shown), except for low birth weight at term in Group 3, in which boys had almost three-fold increased odds ( $P R=2.9 ; 95 \%$ CI: $1.3-$ $6.3)$, and girls had decreased odds $(P R=0.3 ; 95 \%$ CI: $0.03-2.0)$. For 
Table I Characteristics of births of women with breast cancer and of the comparison cohort

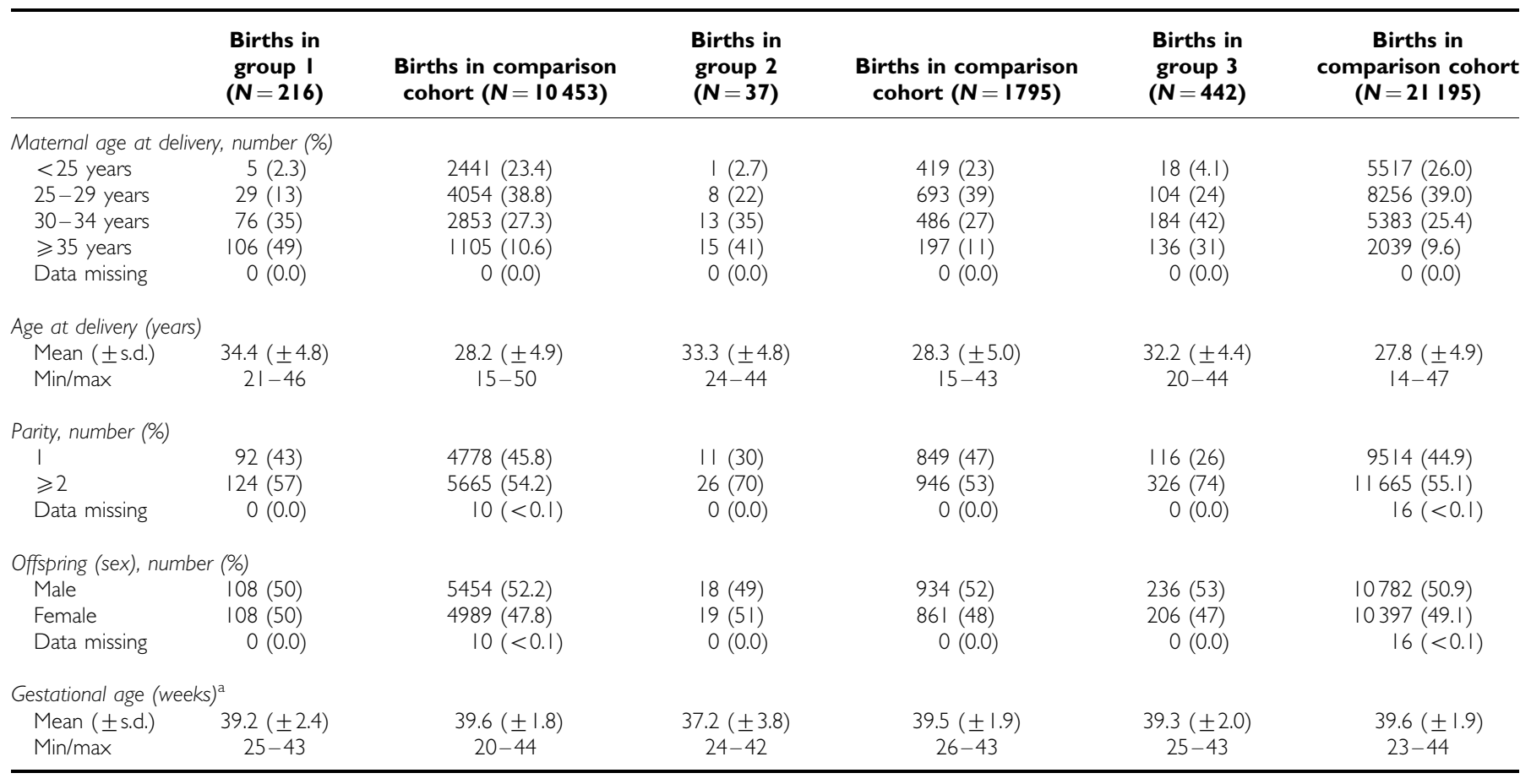

${ }^{a}$ Stillborn babies were excluded from the analyses of mean gestational age. Group I: Births of women diagnosed with breast cancer before pregnancy. Group 2: Births of women diagnosed with breast cancer during pregnancy. Group 3: Births of women diagnosed with breast cancer from the day after giving birth and until 2 years later.

Table 2 Crude and adjusted prevalence odds ratios of birth outcome in women with breast cancer

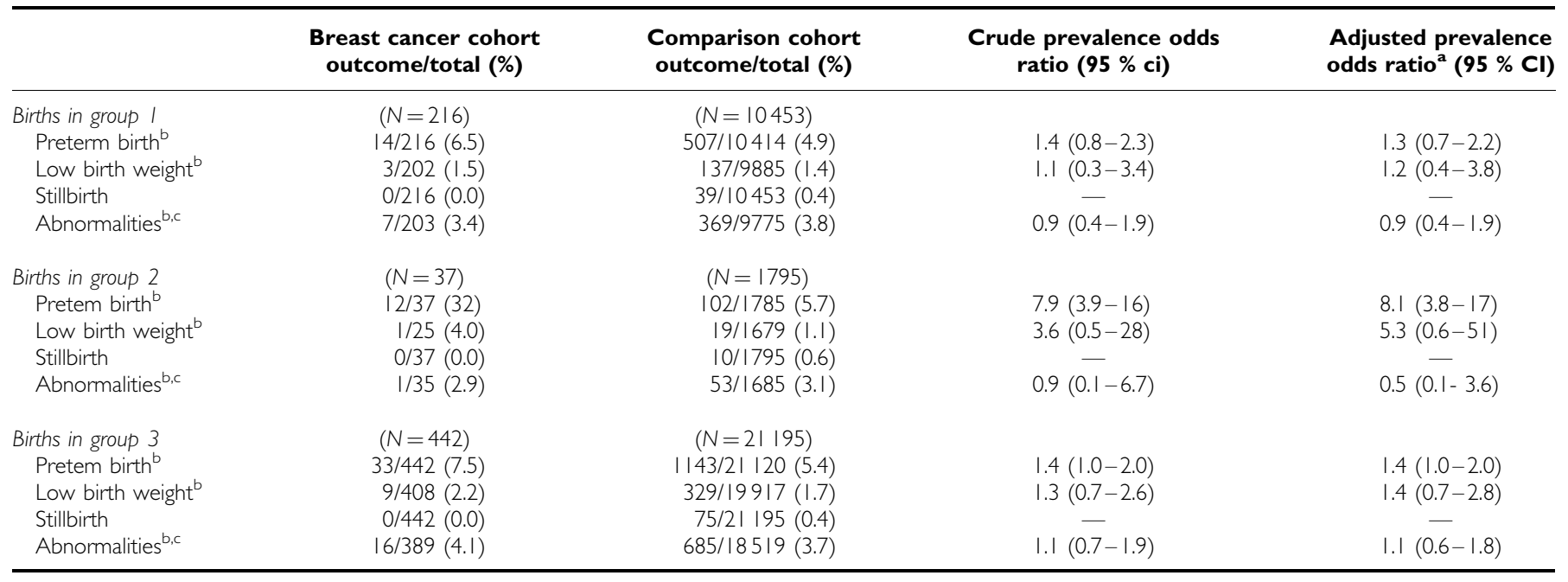

Group I: Birth outcome in women diagnosed with breast cancer before pregnancy. Group 2: Birth outcome in women diagnosed with breast cancer during pregnancy. Group 3:

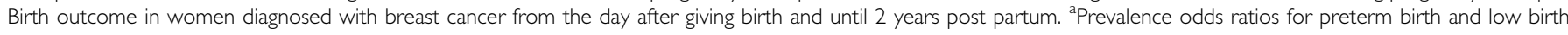
weight at term were adjusted for maternal age ( $<25$ year, 25-29 year, 30-34 year and $\geqslant 35$ year), parity $(1,2+)$ and calendar period of birth (73-86, 87-94, 95-02).

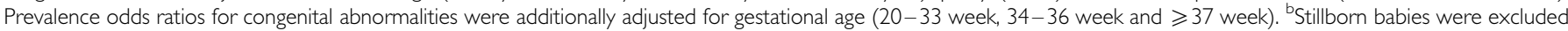
from the analyses of preterm birth, low birth weight at term and congenital abnormalities. 'Data on congenital abnormalities included births from 1977 to 2002.

births in Group 1, stratification according to mother's treatment (surgery alone or other treatment) did not change the overall results (data not shown).

Table 3 shows the adjusted mean differences in birth weight between babies born to women with breast cancer and babies born to comparison mothers. Newborns of women in Groups 1 and 3 had nearly the same mean birth weights as newborns of comparison mothers, whereas newborns of women in Group 2 had a mean birth weight $240 \mathrm{~g}(95 \% \mathrm{CI}=-404 ;-76)$ less than newborns of comparison mothers.

\section{DISCUSSION}

We examined the association between maternal breast cancer and adverse birth outcome in a nationwide cohort and found little difference in the occurrence of preterm birth, low birth weight at term, stillbirth, or congenital abnormalities, compared with the comparison cohort, among newborns of women who were diagnosed with breast cancer before pregnancy.

The eight-fold increased odds of preterm birth for newborns of women who were diagnosed with breast cancer during their 
Table 3 Mean birth weight for newborns of women with breast cancer and for the comparison cohort

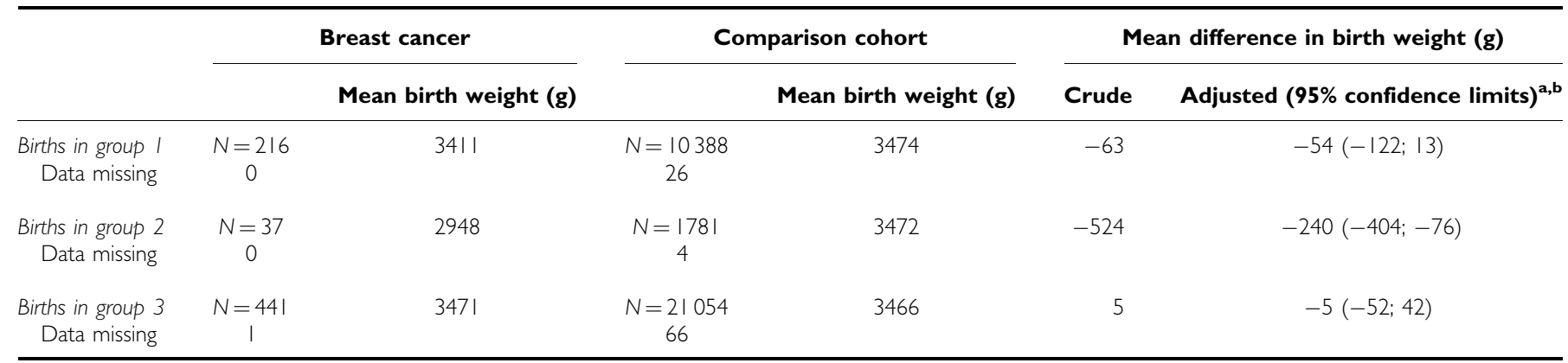

Group I: Mean birth weight for newborns of women with breast cancer before pregnancy. Group 2: Mean birth weight for newborns of women diagnosed with breast cancer

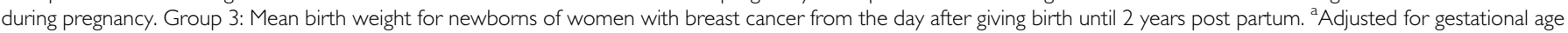
(20-33 week, 34-36 week and $\geqslant 37$ week), mother's age ( $<25$ year, $25-29$ year, 30-34 year, $\geqslant 35$ year), parity (, $2+)$ and calendar period for birth ( $73-86,87-94,95-$

$02)$ in a multivariate regression model. 'btillborn babies were excluded from the analysis.

pregnancy reflected a higher rate of elective early delivery, probably to allow an earlier start of cancer therapy. After adjustment for gestational age, there was a $240 \mathrm{~g}$ reduction in mean birth weight for newborns in this group. The association with preterm birth in Group 3 may be explained by suboptimal intrauterine conditions caused by a preclinical cancer. In this group, only boys had increased odds of low birth weight at term, suggesting that male foetuses are more vulnerable than female.

Our data are derived from a uniformly organized health care system with complete cancer and birth registration. Some selection problems are possible, however. If women with breast cancer had more miscarriages or induced abortions caused by foetal abnormalities than comparison mothers, this phenomenon could explain why we found no increased risk of congenital abnormalities. It has been suggested that exposure to severe periconceptional life events might reduce the male proportion of offspring, partly because of differential abortion of male embryos (Hansen et al, 1999). Thus, a lower proportion of males for offspring of the patients could be an indicator of miscarriages. Another study has indicated an increased risk of miscarriage among women with breast cancer (Velentgas et al, 1999). Our data, however, did not show any important difference in male proportions between the offspring of breast cancer women and offspring of comparison mothers. It has been reported that women with high socioeconomic status have a higher incidence of breast cancer (Danø et al, 2004), while low socioeconomic status has been associated with adverse birth outcome (Luo et al, 2004). We were unable to adjust for socioeconomic status and therefore we may have underestimated the effect of the disease.

A recent study found that treatment data recorded in the Cancer Register are of varying quality (Jensen et al, 2002). However, breast cancer treatment with surgery alone was correctly registered for 95.4\% (Jensen et al, 2002). Coding mistakes are infrequent in the Birth Registry, but data have some misclassifications of gestational age (Kristensen et al, 1996). Our data did not suggest any differential misclassification of preterm birth between women with breast cancer and comparison mothers.

Hospital discharge data are not always coded correctly (Larsen et al, 2003), but Danish data on congenital abnormalities are of high quality compared with other countries, with $80-85 \%$ coded correctly (Larsen et al, 2003). We did not find any clusters of congenital abnormalities in any specific organ system.

Our finding of an increased risk of giving birth preterm for women who were diagnosed with breast cancer during or shortly after pregnancy corroborates the results of two earlier studies (Zemlickis et al, 1992; Smith et al, 2001). In a hospitalbased study, Smith et al (2001) identified 423 cases of breast cancer diagnosed from 9 months preceding delivery until 12 months after delivery over a period of 6 years in California. They reported an odds ratio of $2.2(95 \% \mathrm{CI}=1.7-2.8)$ for prematurity, and an odds ratio of $2.0(95 \% \mathrm{CI}=1.0-4.1)$ for very low birth weight. They adjusted only for maternal age. A hospital-based historical cohort study from 1992 of 118 women, who were pregnant within 9 months before or 3 months after their first treatment for breast cancer, reported a lower mean birth weight after adjustment for gestational age and a higher proportion of preterm births among offspring of women with breast cancer compared with controls (Zemlickis et al, 1992). In these studies, however, the authors did not distinguish between birth outcome of women diagnosed with breast cancer during their pregnancy and women diagnosed shortly after pregnancy. We found a lower mean birth weight limited to newborns of women diagnosed during their pregnancy.

In conclusion, this is the first population-based cohort study of birth outcome in women diagnosed with breast cancer before pregnancy, and the largest cohort study to date of birth outcome in women diagnosed with breast cancer during or shortly after pregnancy. Overall, our results are reassuring regarding the risks of adverse birth outcome for women with breast cancer.

\section{ACKNOWLEDGEMENTS}

The study received financial support from the Danish Cancer Society, the Danish Cancer Research Foundation, the Western Danish Research Forum for Health Sciences and from Ingeborg and Leo Dannins Foundation for Scientific Research.

\section{REFERENCES}

Andersen TF, Madsen M, Jorgensen J, Mellemkjoer L, Olsen JH (1999) The Danish National Hospital Register. A valuable source of data for modern health sciences. Dan Med Bull 46: 263-268
Berry DL, Theriault RL, Holmes FA, Parisi VM, Booser JD, Singletary E, Buzdar AU, Hortobagyi GN (1999) Management of breast cancer during pregnancy using a standardized protocol. J Clin Oncol 17: 855-861 
Blakely LJ, Buzdar AU, Lozada JA, Shullaih SA, Hoy E, Smith TL, Hortobagyi GN (2003) Effects of pregnancy after treatment for breast carcinoma on survival and risk of recurrence. Cancer 100: 465-469, doi:10.1002/cncr.11929

Daly PA, Donnellan P (1992) Breast cancer and pregnancy. Ir Med J 85: $128-130$

Danø H, Hansen KD, Jensen P, Petersen JH, Jacobsen R, Ewertz M, Lynge E (2004) Fertility pattern does not explain social gradient in breast cancer in Denmark. Int J Cancer 111: $451-456$, doi: 10.1002/ijc.20203

Dow KH, Harris JR, Roy C (1994) Pregnancy after breast-conserving surgery and radiation therapy for breast cancer. J Natl Cancer Inst Monogr 16: 131 - 137

Frank L (2000) When an entire country is a cohort. Science 287: 2398-2399, doi:10.1126/science.287.5462.2398

Giacalone PL, Laffargue F, Benos P (1999) Chemotherapy for breast carcinoma during pregnancy: a French national survey. Cancer 86: $2266-2272$

Hansen D, Moller H, Olsen J (1999) Severe periconceptional life events and the sex ratio in offspring: follow up study based on five national registers. BMJ 319: $548-549$

Jensen AR, Overgaard J, Storm HH (2002) Validity of breast cancer in the Danish Cancer Registry. A study based on clinical records from one county in Denmark. Eur J Cancer Prev 11: 359-364

Kelsey JL, Gammon MD, John EM (1993) Reproductive factors and breast cancer. Epidemiol Rev 15: 36-47

Kristensen J, Langhoff-Roos J, Skovgaard LT, Kristensen FB (1996) Validation of the Danish Birth Registration. J Clin Epidemiol 49: 893-897

Kroman N, Jensen MB, Melbye M, Wohlfahrt J, Mouridsen HT (1997) Should women be advised against pregnancy after breast-cancer treatment? Lancet 350: 319-322

Kuerer HM, Gwyn K, Ames FC, Theriault RL (2002) Conservative surgery and chemotherapy for breast carcinoma during pregnancy. Surgery 131: $108-110$

Larsen H, Nielsen GL, Bendsen J, Flint C, Olsen J, Sørensen HT (2003) Predictive value and completeness of the registration of congenital abnormalities in three Danish population-based registries. Scand J Public Health 31: 12 - 16, doi:10.1080/14034940210134194

Luo ZC, Kierans WJ, Wilkins R, Liston RM, Mohamed J, Kramer MS (2004) Disparities in birth outcomes by neighborhood income: Temporal trends in rural and urban areas, British Columbia. Epidemiology 15: 679-686, doi:10.1097/01.ede.0000142149.34095.88

Malamos NA, Stathopoulos GP, Keramopoulos A, Papadiamantis J, Vassilaros S (1996) Pregnancy and offspring after the appearance of breast cancer. Oncology 53: $471-475$

Ribeiro G, Jones DA, Jones M (1986) Carcinoma of the breast associated with pregnancy. Br J Surg 73: 607-609

Ring AE, Smith IE, Jones A, Shannon C, Galani E, Ellis PA (2005) Chemotherapy for breast cancer during pregnancy: an 18-year experience from five London teaching hospitals. J Clin Oncol 23: 4192-4197, doi: $10.1200 /$ JCO.2005.03.038

Smith LH, Dalrymple JL, Leiserowitz GS, Danielsen B, Gilbert WM (2001) Obstetrical deliveries associated with maternal malignancy in California, 1992 through 1997. Am J Obstet Gynecol 184: 1504-1512, doi:10.1067/ mob.2001.114867

Storm HH, Michelsen EV, Clemmensen IH, Pihl J (1997) The Danish Cancer Registry-history, content, quality and use. Dan Med Bull 44: $535-539$

Sutton R, Buzdar AU, Hortobagyi GN (1990) Pregnancy and offspring after adjuvant chemotherapy in breast cancer patients. Cancer 65: $847-850$

Velentgas P, Daling JR, Malone KE, Weiss NS, Williams MA, Self SG, Mueller BA (1999) Pregnancy after breast carcinoma: outcomes and influence on mortality. Cancer 85: 2424-2432

Zemlickis D, Lishner M, Degendorfer P, Panzarella T, Burke B, Sutcliffe SB, Koren G (1992) Maternal and fetal outcome after breast cancer in pregnancy. Am J Obstet Gynecol 166: $781-787$

Zhu JL, Basso O, Hasle H, Winther JF, Olsen JH, Olsen J (2002) Do parents of children with congenital malformations have a higher cancer risk? A nationwide study in Denmark. Br J Cancer 87: 524-528, doi:10.1038/ sj.bjc. 6600488 